\title{
Seroprevalencia a Mycobacterium avium subsp. paratuberculosis en bovinos criollos colombianos Romosinuano y costeño con cuernos
}

\author{
Matiluz Doria R ${ }^{1 *} \bowtie(0)$ M.Sc; Misael Oviedo $\mathrm{P}^{1 \otimes(0)}$ Ph.D; Teresa Oviedo $\mathrm{S}^{2} \bowtie(\mathbb{0}$ Ph.D;

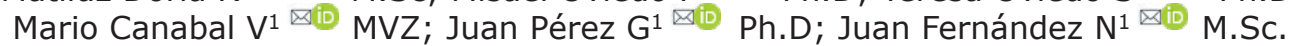

\begin{abstract}
${ }^{1}$ Corporación Colombiana de Investigación Agropecuaria - AGROSAVIA, Colombia.
¿Universidad de Córdoba, Facultad Medicina Veterinaria y Zootecnia, Departamento de Ciencias Pecuarias, Montería, Colombia.

*Correspondencia: mdoriar@agrosavia.co
\end{abstract}

Recibido: Abril 2019; Aceptado: Febrero 2020; Publicado: Julio 2020.

\section{RESUMEN}

Objetivo. Determinar la seroprevalencia y los factores epidemiológicos asociados a Mycobacterium avium subsp Paratuberculosis (MAP) en dos razas de bovinos criollos del centro de investigación AGROSAVIA-Turipaná. Materiales y métodos. Se realizó un estudio epidemiológico de corte transversal. Fueron muestreados 848 bovinos criollos, 403 Romosinuanos y 445 costeño con cuernos (CCC); para el diagnóstico serológico de anticuerpos se utilizó la prueba Elisa Indirecta mediante el kit comercial Parachek ${ }^{\circledR}$ de Prionics. Las variables sexo, edad, raza y tipo de hato fueron los factores epidemiológicos evaluados y correlacionados con la presencia de anticuerpos contra MAP; la asociación estadística fue determinada mediante Odds Ratio y con un modelo multivariado de regresión logística, utilizando un nivel de significancia con $p<0.05$. Resultados. La seroprevalencia general de los bovinos criollos a MAP fue de $2.35 \%$ (IC 95\%, 1.34-3.38); sin embargo, en los Romosinuano fue de $0.74 \%$ y en los CCC fue de $3.82 \%$, siendo las diferencias estadísticamente significativas (valor $\mathrm{p}=0.003$ ). También, empleando un análisis univariado, fueron más afectados las hembras y los animales mayores a un año. El análisis multivariado identificó como factores epidemiológicos las variables raza y sexo. Conclusiones. En las razas criollas del centro de investigación AGROSAVIATuripaná, la seroprevalencia a MAP fue baja; sin embargo, en la raza CCC el riesgo de contraer la enfermedad es seis veces mayor con relación a la raza Romosinuano. Más aún, se pudo evidenciar que las hembras tienen mayor riesgo de adquirir la enfermedad.

Palabras clave: Agrosavia; diagnóstico serológico; enfermedad de Johne; enteropatía; epidemiología; factores de riesgo (Fuentes: DeCS, FAO).

\section{ABSTRACT}

Objective. Determine the seroprevalence and epidemiological factors associated with Mycobacterium avium subsp. paratuberculosis (MAP) in two Creole cattle breeds of the Turipaná research center AGROSAVIA. Materials and methods. A cross-sectional epidemiological study was conducted, a total of 848 Creole bovine animals were sampled, 403 Romosinuano and 445 costeño con cuernos (CCC);

Como citar (Vancouver).

Doria RM, Oviedo PM, Oviedo ST, Canabal VM, Pérez GJ, Fernández NJ. Seroprevalencia a Mycobacterium avium subsp. paratuberculosis en bovinos criollos colombianos Romosinuano y costeño con cuernos. Rev MVZ Cordoba. 2020; 25(2):e1611. https://doi.org/10.21897/rmvz.1611 
for the serological diagnosis of antibodies, the Elisa Indirect test was used with the commercial kit Parachek ${ }^{\circledR} 2$ by Prionics. The variables sex, age, breed and herd type were the epidemiological factors evaluated and correlated with the presence of antibodies against MAP; the statistical association was established using the Odds Ratio and a multivariate logistic regression model, employing a significance level with $\mathrm{p}<0.05$. Results. The general seroprevalence of the Creole cattle to MAP was $2.35 \%$ (95\% CI, 1.34-3.38); however, in the Romosinuano it was $0.74 \%$ and in the CCC it was $3.82 \%$, being this difference statistically significant $(p=0.003)$. Furthermore, employing a univariate way analysis, females and animals older than one year of age were more affected. The multivariate analysis identified the breed and sex variables as epidemiological factors. Conclusions. In the Creole breeds of the AGROSAVIA-Turipaná research center, MAP seroprevalence was low; however, in the Costeño Con Cuernos breed, the risk of contracting the disease is six times higher than in the Romosinuano breed. Moreover, it was shown that females have a higher risk of acquiring the disease.

Keywords: Agrosavia; serological diagnosis; Johne's disease; enteropathy; epidemiology; risk factors (Sources: DeCS, FAO).

\section{INTRODUCCIÓN}

La paratuberculosis o enfermedad de Johne, se caracteriza por una inflamación crónica granulomatosa del tracto digestivo que afecta principalmente a los rumiantes domésticos. Su manifestación clínica es una enteropatía con pérdida de proteínas que causa diarrea, emaciación, hipoproteinemia y en algunos casos la muerte (1), el agente causal es el Mycobacterium avium, subespecie paratuberculosis (MAP) (2). En los bovinos jóvenes la transmisión temprana se da por la vía oro-fecal por ingestión de alimentos, leche, y contacto con áreas contaminadas (3).

La infección por MAP se da en los primeros meses de vida del animal, después de un largo periodo de incubación se manifiestan los signos clínicos que surgen entre los dos y cinco años (4).

La paratuberculosis bovina causa grandes pérdidas económicas debido a los efectos negativos sobre la salud de los animales, producción y reproducción (5). Es una enfermedad de declaración obligatoria y debe ser notificada a la Organización Mundial de Sanidad Animal - OIE (6). Para un control adecuado se debe identificar el estado de infección de los hatos, las rutas de transmisión y las diferentes fuentes de infección (3).

Dentro de las pruebas para detección de anticuerpos contra MAP, la técnica de ELISA es la de mayor disponibilidad y ampliamente utilizada, aunque presenta sensibilidad baja, especialmente en las primeras etapas de la enfermedad (7). Con relación al diagnóstico del agente causal, éste presenta un largo periodo de espera debido a un prolongado período de incubación y riesgos de contaminación (8).

En Suramérica y el Caribe se ha estimado que la prevalencia general de la enfermedad en el ganado bovino es de $16,9 \%$ y a nivel de hatos del $75.8 \%$ (9). En Colombia la paratuberculosis bovina fue diagnosticada por primera vez en el año 1924; años más tarde fueron reportados otros casos en el departamento de Meta por el Instituto Colombiano Agropecuario (ICA) (10).

A pesar de que se ha confirmado la circulación de MAP en Colombia desde hace muchos años, los estudios realizados son insuficientes para evidenciar su magnitud, no obstante, el número investigaciones originales realizadas en la última década sugiere un creciente interés por este microorganismo en el país (11).

En Colombia existen varios bancos de germoplasma de bovinos criollos, destinados a la conservación de la diversidad genética; sin embargo, existen pocos estudios publicados de prevalencia de MAP en estos repositorios de ganado criollo colombiano (12); por lo cual el objetivo de este estudio fue determinar la seroprevalencia y los factores epidemiológicos asociados a Mycobacterium avium subespecie paratuberculosis (MAP) en los bovinos criollos del centro de investigación Turipaná-AGROSAVIA.

\section{MATERIALES Y MÉTODOS}

Sitio de estudio. El estudio se desarrolló en el centro de investigación Turipaná, de AGROSAVIA; localizado entre las latitudes $8^{\circ} 51^{\prime} 50^{\prime \prime}$ y $8^{\circ} 49^{\prime} 40^{\prime \prime}$ 
y las longitudes -75040'30" y -75046'10", en el municipio de Cereté, departamento de Córdoba, Colombia. Clasificado como bosque seco tropical, con altitud de $14 \mathrm{msnm}$, temperatura promedio de $27.5^{\circ} \mathrm{C}, 81 \%$ de humedad relativa y precipitación promedio anual de $1.340 \mathrm{~mm}$; durante el año presenta un período de sequía y otro de lluvias, el período de lluvias va entre abril a noviembre.

Tipo de estudio y tamaño de la muestra. Se realizó un estudio de corte transversal, siendo evaluados todos los bovinos de las razas criollas Romosinuano (403 animales) y Costeño Con Cuernos (445 animales) del centro de investigación Turipaná durante los meses de mayo a octubre del año 2016. La edad de los animales muestreados osciló entre un mes y 15 años; 160 menores a seis meses, 42 entre seis y 11 meses y 616 de un año en adelante.

Toma, procesamiento y análisis de las muestras. Previa desinfección del área, se colectaron $5 \mathrm{ml}$ de sangre en la vena coccígea por cada animal en tubos tipo vacutainer sin anticoagulante. Las muestras fueron identificadas y conservadas a una temperatura de $4^{\circ} \mathrm{C}$ durante 4 horas aproximadamente hasta la obtención del suero. Para ello las muestras fueron centrifugadas a $3500 \mathrm{rpm}$ durante 5 minutos, posteriormente depositadas en viales y conservadas a temperatura de $-20^{\circ} \mathrm{C}$ hasta su análisis en el laboratorio del centro de investigación Tibaitatá de AGROSAVIA, en el departamento de Cundinamarca. El análisis fue realizado mediante prueba de ELISA INDIRECTA utilizando el kit de diagnóstico comercial Parachek ${ }^{\circledR} 2$ Prionics AG. según recomendaciones del fabricante.
Análisis estadístico. El estudio de seroprevalencia se acompañó de una encuesta epidemiológica encaminada a determinar factores de riesgo que podrían tener relación con la patología en estudio; sexo, raza (Romosinuano y CCC), edad (menores de un año y mayores de un año) y hato (Banco de germoplasma y Mejoramiento genético) fueron factores investigados. Estos factores fueron asociados de forma univariada con los resultados diagnósticos; se determinó como medida de efecto la Odds Ratio (OR) y se confirmó mediante el estadístico Chi-cuadrado o con la prueba exacta de Fisher con un nivel de significancia de 0.05 . Finalmente, se construyó un modelo de regresión logística multivariada para explicar la correlación entre los factores y la seroprevalencia de paratuberculosis; las variables incluidas en el modelo fueron preseleccionadas mediante el análisis univariado, incluyendo las que presentaron $p<0.2$. Los datos fueron analizados en el software EpiInfo 7.2.1.0.

\section{RESULTADOS}

De las 848 muestras analizadas, se encontraron anticuerpos MAP en 20 animales indicando una seroprevalencia aparente de $2.35 \%$ (IC 95\%, 1.34-3.38) para las razas bovinas criollas del centro de investigación Turipaná.

Análisis univariado. La tabla 1 muestra el análisis univariado de las variables estudiadas, observándose que solamente sexo y raza presentaron asociación estadística con la seroprevalencia de MAP.

Tabla 1. Análisis univariado de factores epidemiológicos asociados a seroprevalencia de MAP en bovinos criollos del centro de investigación Turipaná - AGROSAVIA.

\begin{tabular}{|c|c|c|c|c|c|c|c|c|}
\hline \multirow{2}{*}{ Variables } & \multirow{2}{*}{ Categorías } & \multirow{2}{*}{$n+$} & \multirow{2}{*}{ n- } & \multirow{2}{*}{ Seroprevalencia } & \multirow{2}{*}{ P valor } & \multirow{2}{*}{ O.R } & \multicolumn{2}{|c|}{ IC } \\
\hline & & & & & & & Inferior & Superior \\
\hline \multirow[b]{2}{*}{ Raza } & Romosinuano & 3 & 400 & $0.74 \%$ & \multirow{2}{*}{0.003} & \multirow{2}{*}{5.296} & \multirow{2}{*}{1.540} & \multirow{2}{*}{18.201} \\
\hline & $\mathrm{CCC}$ & 17 & 428 & $3.82 \%$ & & & & \\
\hline \multirow{2}{*}{ Sexo } & Macho & 2 & 271 & $0.73 \%$ & \multirow{2}{*}{0.032} & \multirow{2}{*}{4.378} & \multirow{2}{*}{1.008} & \multirow{2}{*}{19.002} \\
\hline & Hembra & 18 & 557 & $3.13 \%$ & & & & \\
\hline \multirow{2}{*}{ Edad } & $\leq 1$ año & 2 & 230 & $0.86 \%$ & \multirow{2}{*}{0.078} & \multirow{2}{*}{3.461} & \multirow{2}{*}{0.797} & \multirow{2}{*}{15.041} \\
\hline & $>1$ año & 18 & 598 & $2.92 \%$ & & & & \\
\hline \multirow{3}{*}{ Hato } & $\begin{array}{c}\text { Mejoramiento } \\
\text { Genético }\end{array}$ & 1 & 81 & $1.22 \%$ & \multirow{3}{*}{0.475} & \multirow{3}{*}{2.060} & \multirow{3}{*}{0.272} & \multirow{3}{*}{15.593} \\
\hline & Banco & & & & & & & \\
\hline & Germoplasma & 19 & 747 & $2.48 \%$ & & & & \\
\hline
\end{tabular}


Análisis multivariado. Teniendo en cuenta el modelo de regresión logística (Tabla 2), las variables sexo y raza mantuvieron su significancia estadística; por lo tanto, los animales de raza CCC tienen 5.6 veces mayor susceptibilidad de ser seropositivos a MAP y las hembras bovinas criollas tiene 4.7 veces más positividad que los machos criollos.

Tabla 2. Modelo de regresión logística para MAP en el hato bovino criollo de AGROSAVIA Turipaná.

\begin{tabular}{ccccccc}
\hline \multirow{2}{*}{ Variables Categorías } & P-valor & O.R & \multicolumn{2}{c}{ IC 95\% } \\
\hline \multirow{2}{*}{ Sexo } & $\begin{array}{c}\text { Macho } \\
\text { Hembra }\end{array}$ & 0.037 & 4.75 & 1.091 & 20.710 \\
\multirow{2}{*}{ Raza } & $\begin{array}{c}\text { Romosinuano } \\
\text { CCC }\end{array}$ & 0.006 & 5.60 & 1.627 & 19.328 \\
\hline
\end{tabular}

\section{DISCUSIÓN}

La seroprevalencia encontrada en este estudio $(2.35 \%)$ en razas bovinas criollas del centro de investigación Turipaná fue similar a la encontrada en hatos lecheros del departamento de Antioquia (2\%) usando el mismo kit de ELISA (Parachek ${ }^{\circledR 2}$ Prionics AG.) (13). Por otra parte, Vélez et al (12) en un estudio realizado en ganado criollo Blanco Orejinegro no encontraron seroprevalencia para esta enfermedad mediante técnica de ELISA.

En el departamento de Córdoba, en el año 2010, se detectó una seroprevalencia del $25 \%$ en vacas lecheras (14), recientemente Caraballo et al (15) determinaron la presencia de MAP en heces de bovinos en el departamento de Sucre, visualizando el Mycobacterium en el $21.15 \%$ del total de muestras analizadas, otro estudio en ganadería de carne en el municipio de Caucasia mostraron que el $33.8 \%(n=151)$ de los bovinos estudiados fueron seropositivos a MAP (13); en ganadería de leche en el departamento de Nariño, $8 \%$ de los animales $(n=958)$ fueron seropositivos (16). Estas seroprevalencias fueron mayores a las encontradas en este estudio, sin embargo, ninguna supera los valores encontrados en 2006 en ganadería doble propósito, en Venezuela, donde se detectó que el $72.1 \%(n=240)$ de los bovinos fueron seroprevalentes (17).

Variable raza. Algunos estudios demuestran baja prevalencia de MAP en razas criollas. $1.45 \%$ de seropositividad $(n=207)$ fue determinada en la raza Limonero (8) y $0 \%$ en animales Blanco Orejinegro (12); en este último estudio, se observó seropositividad en los animales con cruces con genética Brahman. Aunque en el presente estudio, la prevalencia de MAP fue baja en las dos razas, ante situaciones similares de población, manejo y alimentación, fue evidenciada una menor susceptibilidad del Romosinuano que podría ser explicada por posibles diferencias genéticas. Los animales de la raza CCC fueron 5.3 OR más afectados con relación a los Romosinuanos; sin embargo, por ser el primer estudio de paratuberculosis en estas dos razas se requiere realizar otros trabajos que profundicen la mayor susceptibilidad para la raza CCC.

Variable sexo. El análisis univariado de esta variable determinó que las hembras fueron 4.37 OR más afectadas que los machos. la mayor susceptibilidad de las hembras podría ser atribuida a las alteraciones inmunológicas y al estrés ocasionado durante el parto y la lactancia (18).

Variable edad. La mayor prevalencia se presentó en los animales mayores de un año, con el $2.92 \%$ de seropositividad, sin embargo, no se encontró diferencia estadística entre los dos grupos etarios y la seropositividad de MAP. Resultados similares fueron observados en bovinos estudiados en Caucasia, en el nordeste de Antioquia (12) y en el sur de Nariño (16).

Cuando se estudia paratuberculosis teniendo en cuenta la variable edad, la especificidad del ensayo se convierte en un reto; en ganado de leche se ha demostrado, que el tiempo trascurrido desde la infección, generalmente en un ternero, hasta la enfermedad clínica puede variar de uno hasta 12 años, (19); adicionalmente, el serodiagnóstico suele ser negativo en animales menores de un año debido a que durante las primeras etapas de la enfermedad, predomina la respuesta inmune mediada por células, mientras que la respuesta de anticuerpos séricos es mínima o ausente (20).

Teniendo en cuenta lo anterior, se puede considerar que las pruebas serológicas como ELISA, realizadas una única vez, no son capaces de identificar la enfermedad subclínica en todos los animales, por lo que se sugiere su realización periódica para detectar animales infectados en el hato antes de la manifestación clínica.

Variable hato. Las condiciones de manejo y alimentación fueron similares entre los hatos Banco de Germoplasma y el Programa Mejoramiento Genético; la diferencia entre los dos hatos está relacionada con la finalidad; el Banco de Germoplasma es repositorio para la conservación de la diversidad genética y el Programa de Mejoramiento Genético tiene uso estratégico para el aprovechamiento de las ventajas de la raza. La prueba exacta de Fisher no arrojó asociación estadística entre los dos hatos; sin embargo, el 95\% (19/20) 
de los animales positivos fueron encontrados en el Banco de Germoplasma, situación que pudo ser influenciada por agrupar el $90 \%$ de los animales del estudio, lo que posibilita una mayor trasmisión. En otro estudio se ha identificado que el tamaño del hato es un factor significativo para la propagación y la prevalencia de MAP (21), por lo cual se deben implementar medidas de bioseguridad internas para disminuir la exposición de la enfermedad según las características de cada hato (22).

Los ensayos serológicos son ampliamente utilizados para estimar prevalencias de MAP en los rebaños, sin embargo, la baja sensibilidad de estas pruebas puede dar una cantidad sustancial de resultados falsos negativos (23); la prueba utilizada en este estudio reporta en bovinos $99 \%$ de especificidad y hasta $80 \%$ de sensibilidad antes de la aparición de signos clínicos. Debido a la baja prevalencia encontrada en este estudio, el número de falsos negativos no incrementaría de forma considerable la prevalencia verdadera del rebaño; se proyecta la realización de nuevos estudios principalmente en los animales que fueron menores a un año.
Cabe anotar que, en el modelo de regresión logística, las variables sexo y raza mantuvieron su significancia estadística.

Este es el primer estudio de paratuberculosis bovina que se realiza en Colombia para las razas criollas Romosinuano y CCC. En conclusión, aunque se reportó una seroprevalencia relativamente baja para MAP en las dos razas criollas del Centro de Investigación Turipaná, se requiere implementar medidas de control y prevención con el fin de reducir los factores de riesgo identificados y asociados a esta enfermedad.

\section{Conflicto de intereses}

Con relación a este artículo, ninguno de los autores tiene conflicto de intereses.

\section{Agradecimientos}

Los autores agradecen al Sistema de Bancos de Germoplasma de la Nación para la Alimentación y la Agricultura (SBGNAA) con sede en el C.I. Turipaná de AGROSAVIA, por permitir el acceso a los bovinos utilizados en esta investigación.

\section{REFERENCIAS}

1. Sweeney RW, Collins MT, Koets AP, McGuirk SM, Roussel AJ. Paratuberculosis (Johne's disease) in cattle and other susceptible species. J Vet Intern Med. 2012; 26(6):1239-1250. https://doi.org/10.1111/ j.19391676.2012.01019.x

2. Sigurðardóttir ÓG, Valheim M, Press CM. Establishment of Mycobacterium avium subsp. paratuberculosis infection in the intestine of ruminants. Adv Drug Deliv Rev. 2004; 56(6):819-834. https://doi. org/10.1016/j.addr.2003.10.032

3. Harris NB, Barletta RG. Mycobacterium avium subsp. Paratuberculosis in veterinary medicine. Clin Microbiol Rev. 2001; 14(3):489-512. https://doi.org/10.1128/ CMR.14.3.489-512

4. Kennedy DJ, Benedictus G. Control of Mycobacterium avium subsp. paratuberculosis infection in agricultural species. Rev Sci Tech. 2001; 20(1):151-179. https://doi.org/10.20506/rst.20.1.1274
5. Zapata RM, Rodas GJ, Maldonado EJ. Paratuberculosis bovina: ¿conocemos la situación real de la enfermedad en la ganadería colombiana? Rev Colom Cienc Pecua. 2008; 21(3):420-435. https:// aprendeenlinea.udea.edu.co/revistas/index. $\mathrm{php} / \mathrm{rccp} / \mathrm{article} / \mathrm{view} / 324313$

6. OMS. Paratuberculosis. [en línea]. Organización Mundial de Sanidad Animal: París; 2019. URL disponible en: http:// www.oie.int/es/sanidad-animal-en-elmundo/enfermedades-de-Ios-animales/ paratuberculosis/

7. O'Mahony Jim, HILL Colin. Rapid realtime PCR assay for detection and quantitation of Mycobacterium avium subsp. paratuberculosis DNA in artificially contaminated milk. Appl Environ Microbiol. 2004; 70(8):4561-4568. https://doi. org/10.1128/AEM.70.8.4561-4568.2004 
8. Sánchez-Villalobos A, Arráiz-Rodríguez $\mathrm{N}$, Becerra-Ramírez L, Faria N, MonteroUrdaneta M, Oviedo-Bustos A et al. Infección por mycobacterium avium subsp. Paratuberculosis en un rebaño criollo limonero. Rev Cient. (Maracaibo). 2009; 19(6):555-565. http://www.saber.ula. ve/bitstream/handle/123456789/29699/ articulo1. pdf? sequence $=1$ \&isAllowed $=y$

9. Fernández-Silva JA, Correa-Valencia NM, Ramíre NF. Systematic review of the prevalence of paratuberculosis in cattle, sheep, and goats in Latin America and the Caribbean. Trop Anim Health Prod. 2014; 46(8):1321-1340. https://doi.org/10.1007/ $\underline{\text { s11250-014-0656-8 }}$

10. Espeschit IF, Schwarz DGG, Faria ACS, Souza MCC, Paolicchi FA, Juste RA, et al. Paratuberculosis in Latin America: a systematic review. Trop Anim Health Pro. 2017; 49(8):1557-1576. https://doi. org/10.1007/s11250-017-1385-6

11. Correa-Valencia N, García-Tamayo YM, Fernández-Silva JA. (Mycobacterium avium subsp. paratuberculosis) in Colombia, 1924-2016. Rev Colom Cienc Pecua. 2018; 31(3):165-179. https://doi.org/10.17533/ udea.rccp.v31n3a01

12. Vélez AM, Rendón DY, Valencia RA, Ramírez VN, Fernández-S J. Seroprevalencia de Mycobacterium avium Subsp. Paratuberculosis (MAP) en una granja de ganado de carne de bosque húmedo tropical en Caucasia, Antioquia, Colombia. Rev Colombiana Cienc Anim - Recia. 2016; 8(2):167-176. https://doi.org/10.24188/ recia.v8.n2.2016.184

13. Correa-Valencia NM, Ramírez NF, Olivera $M$, Fernández-Silva JA. Milk yield and lactation stage are associated with positive results to ELISA for Mycobacterium avium subsp. paratuberculosisin dairy cows from Northern Antioquia, Colombia: a preliminary study. Trop Anim Health Prod. 2016; 48(6):11911200. https://doi.org/10.1007/s11250$\underline{016-1074-x}$

14. De Waard JH. ¿Ordeñando micobacterias del ganado? Impacto económico y en salud de Tuberculosis bovina y Paratuberculosis en Colombia? Rev MVZ Córdoba. 2010；15(2):2037-2040. https://doi. org/10.21897/rmvz.998

15. Caraballo-Blanco Libardo, Castellar-Martínez
Anais, Pardo-Pérez Enrique. Mycobacterium avium subespecie paratuberculosis en heces de bovinos del municipio de Sincelejo, Sucre, Colombia. Rev. investig. vet. Perú. 2018; 29(3):987-995. http://dx.doi. org/10.15381/rivep.v29i3.14111.

16. Benavides BB, Cadena Á VA, Misnaza CAM. Estudio epidemiológico de paratuberculosis bovina en hatos lecheros del sur de Nariño, Colombia. Rev Med Vet. 2016; (31):57-66. https://doi.org/10.19052/mv.3709

17. Coromoto A, de Rolo M, Clavijo A, Valle A. Caracterización de la paratuberculosis bovina en ganado doble propósito de los Ilanos de Monagas, Venezuela. Zootec Trop. 2006; 24(3):321-332. http://www.bioline. org.br/abstract?zt06026

18. Feola RP, Collins MT,Czuprynski CJ. Hormonal modulation of phagocytosis and intracellular growth of Mycobacterium avium s,s, paratuberculosis in bovine peripheral blood monocytes. Microb Pathogen. 1999; 26(1):1-11. https://doi.org/10.1006/ mpat.1998.0246

19. Jubb T, J Galvin. Herd testing to control bovine Johne's disease. Vet. Microbiol. 2000; 77(3-4):423-428. https://doi.org/10.1016/ $\underline{\mathrm{S} 0378-1135(00) 00327-8}$

20. Maroudam V, Mohana Subramanian B, Praveen Kumar P, Dhinakar Raj G. Paratuberculosis: Diagnostic Methods and their Constraints. J Veterinar Sci Technol. 2015; 6(5):1000259 https://doi. org/10.4172/2157-7579.1000259

21. Barrett DJ, Mee JF, Mullowney P, Good M,McGrath G,Clegg T, More SJ. Risk factors associated with Johne's disease test status in dairy herds in Ireland. Vet Rec. 2011; 168(15):410. http://dx.doi.org/10.1136/ vr.c6866

22. Collins MT, Eggleston V, Manning EJB. Successful control of Johne's disease in nine dairy herds: results of a six-year field trial. J Dairy Sci. 2010; 93(4):1638-1643. https:// doi.org/10.3168/jds.2009-2664

23. Lilenbaum W, Marassi CD, Oelemann WMR. Paratuberculosis: An Update. Braz J Microbiol. 2007; 38(4):580-590. http://dx.doi. org/10.1590/S1517-83822007000400001 